\title{
ION IRRADIATION INDUCED EFFECTS ON AIRLESS BODIES IN THE SOLAR SYSTEM
}

\author{
G. Strazzulla ${ }^{1}$
}

\begin{abstract}
Irradiation by cosmic ions plays a relevant role in the "space weathering" of solid materials in the airless bodies (rocky and/or icy objects) in the Solar System. Here, I discuss about some of the effects that have been studied in "simulation" experiments with a view to their astrophysical relevance. In particular I review some experimental results obtained so far after irradiation of relevant materials, namely: sputtering, ion implantation, and chemical synthesis of molecular species different from the original ones. All of those effects are accomplished by changes in the optical properties of irradiated layers and then of the color of the surfaces of the airless bodies. Some specific examples of the application of the experimental results to the physics and chemistry of planetary surfaces are discussed as well.
\end{abstract}

\section{Introduction}

There are several classes of airless bodies in the Solar System. Roughly they can be separated in rocky and icy objects. The former include different populations such as Main Belt Asteroids, Near Earth Objects (NEO), Mercury and the Moon. The icy (mostly dominated by solid water) objects include the moons of the outer planets, Pluto, the Trans Neptunian Objects (TNO) and Comets. The surfaces of these objects, because of the lack of substantial atmospheres, are exposed to external radiations (photons and particles).

The alteration induced by photolysis and radiolysis (often referred to as "space weathering") is considered of primary relevance in understanding the physicochemical processes governing the evolution of, at least, the external layers (those from which the solar electromagnetic radiation is reflected) of the different objects. Progress in the knowledge of those processes is based on laboratory experiments whose results, some times used as input parameters for models, allow to understand observations and suggest new space and/or Earth based enterprises.

${ }^{1}$ INAF-Istituto Nazionale di Astrofisica - Osservatorio Astrofisico di Catania, via S. Sofia 78, 95123 Catania, Italy; e-mail: gianni@oact.inaf.it; http://web.ct.astro.it/weblab/ 


\section{Laboratory vs. Space}

In the several laboratories active in the field in Europa, USA and Japan, a scattering chamber is faced to an ion beam and, in most of them, it is also faced, through transparent windows, to spectrophotometers (UV-Vis, NIR or IR) that allow to obtain transmittance or reflectance spectra of the chosen targets. High or ultrahigh vacuum chambers are used. The rocky targets are directly placed in the chamber. Frosts are accreted from the gas phase onto a transparent (KBr, CsI, silicon, etc.) or reflecting (alluminum, gold, etc.) substrate placed in contact with a cold finger (10-300 K). Among the techniques used by different groups to characterize the ion induced effects, in addition to UV-Vis-NIR-IR (transmittance and reflectance) spectroscopy, there are mass spectrometry, thermal programmed desorption and Raman spectroscopy.

Fast ions penetrating solids loose energy by elastic collision with target nuclei (nuclear stopping, $\mathrm{S}_{n}$ ) and by inelastic ionizations and excitation (electronic stopping, $\mathrm{S}_{e}$ ). The percentage of energy lost in elastic or inelastic collision depends on the ion mass and energy. For practical experimental reasons it is often not possible to use the most appropriate ion and energy to "simulate" a given astrophysical environment. Thus, as an example, if experiments are performed that are mostly aimed at simulating the effects of the solar wind protons (that is at energy of about $1 \mathrm{keV}$ corresponding to $\mathrm{S}_{n} / \mathrm{S}_{e}$ of about 0.23 ), it is more appropriate to use ions and energies such that $\mathrm{S}_{n} / \mathrm{S}_{e}$ is on the order of $0.1-1$ (e.g. Ar ions at $0.2-2 \mathrm{MeV}$ ), rather than protons at, say, $100 \mathrm{keV}$ having $\mathrm{S}_{n} / \mathrm{S}_{e}=1.8 \times 10^{-3}$. [Calculation have been done by using the SRIM (Zeigler et al. 2008) software].

The experimental results have to be applied with caution to the different astrophysical environments. In fact in laboratory "simulations" some relevant parameters are reproduced very well but others cannot be reproduced at all. As an example, reproducing the total fluence (ion $\mathrm{cm}^{-2}$ ) expected on a given planetary object is easy. But even though the laboratory ion currents (ion $\mathrm{cm}^{-2} \mathrm{~s}^{-1}$ ) are maintained low enough to avoid collective effects (e.g. macroscopic heating of the target) they are many orders of magnitude greater than in space. Therefore any result that depends on the dose rate cannot be applied to astrophysical scenarios.

\section{Results}

\subsection{Sputtering}

Most (all?) of the minor objects in the Solar System have tenuous atmospheres most of which being not gravitationally bounded, they require a continuous supply of atoms/molecules from the surface. Several mechanisms have been suggested as potentially relevant to remove material from the surface: photo- and thermal desorption, meteoritic impact, geological (geyser-like) activity and ion sputtering (e.g., Johnson 2002). Species released from the surfaces have velocity distributions that are characteristic of the ejecting process. Sputtering from keV-MeV ions, such that of solar wind or of the ions in the magnetospheres of the giant planets, produce 
species that have energies from $0.2 \mathrm{eV}$ to some hundred $\mathrm{eV}$, peaking at few $\mathrm{eV}$. The ejected atoms and molecules, can escape the gravitational field, or return to the surface after a ballistic trajectory. If the object has a magnetosphere, as it is the case of e.g. Mercury, the released species can be photo-ionized and picked up thus contributing to the ion population of that planet.

The - still scarce - laboratory experiments on sputtering of silicates by low energy ions (about $1 \mathrm{keV} / \mathrm{u}$, simulating solar wind impacts) are however particularly relevant to understand the composition of the exospheres of Mercury and the Moon or also, through a modeling of astronomical data, the surface composition of those objects (e.g. Milillo et al. 2011). Thus it seems relevant to the laboratory research by Dukes et al. (2011) that have recently irradiated with $4 \mathrm{keV}$ $\mathrm{He}^{+}$ions, both $\mathrm{Na}$ bearing tectosilicates (albite, labradorite, and anorthoclase) and adsorbed $\mathrm{Na}$ layers deposited on albite and olivine. They measured a cross section for sputtering of $\mathrm{Na}$ adsorbed on mineral surfaces and demonstrated that a large fraction of the $\mathrm{Na}$ is sputtered as ions rather than as neutral atoms. The results could be relevant, as an example, to model the Mercury's exosphere. It is in fact known that neutral sodium abundance strongly increases at high latitudes where magnetic field lines allow solar wind ions to directly impact on the surface (e.g. Vervack et al. 2010).

Much more data are available, since the seminal paper by Brown et al. (1978), for the sputtering of ices for which the total electronic sputtering yield $\mathrm{Y}$ is proportional to the square of the electronic-stopping power. The early laboratory results have been used to predict the principal atmospheric component of Europa, $\mathrm{O}_{2}$, and its average column density (Johnson et al. 1998). Since then many results have been obtained and applied both to calculate the lifetimes, against sputtering, for the destruction of icy grains in astrophysical environments and to understand the interaction between surface and atmospheres (see e.g. Baragiola 2004).

\subsection{Chemical alteration}

The large majority of experiments of ion irradiation have been and are performed with the aim to investigate the induced non-thermal, "hot" chemical reactions that lead to the formation of a large number of molecules, from the simplest to very complex, not present in the target before irradiation. These molecules include long-chain refractory organic materials that are among the best candidate to explain the colors of the minor objects in the outer Solar System. Frozen layers made of simple molecules, pure (e.g. $\mathrm{H}_{2} \mathrm{O}, \mathrm{CH}_{4}, \mathrm{NH}_{3}, \mathrm{CO}_{2}, \mathrm{CO}, \mathrm{SO}_{2}$ and many others) or mixed, are deposited at low temperatures $(10-150 \mathrm{~K})$. If the thickness of the target is thin with respect to the ion penetration depth, the ion travels through the target, deposits part of its energy and leaves the target. This kind of experiments is appropriate to simulate icy mantles on grains in the interstellar medium bombarded by cosmic energetic ions and we refer to them as irradiation experiments. For a review of a large numbers of experiments with a particular view to their applications to TNOs, see Hudson et al. (2008). 
When the thickness of the target is greater than the ion penetration depth, the ions are implanted in the target and we refer to as implantation experiments. Implanted reactive ions $\left(e . g ., \mathrm{H}^{+}, \mathrm{C}^{+}, \mathrm{N}^{+}, \mathrm{O}^{+}, \mathrm{S}^{+}\right)$induce all of the effects of any other ion, but in addition have a chance, being stopped in the target, to form new species containing the projectile. These experiments are of fundamental relevance to clarify the origin of the minor species observed in the icy moons of the outer Solar System. A still open question is whether those minor species are endogenic i.e. native from the satellite or are produced by exogenic processes.

In fact although water ice is by far the dominant species, minor features have been observed in the near IR spectra of the satellites and attributed to C-H, $\mathrm{H}_{2} \mathrm{O}_{2}$, $\mathrm{S}-\mathrm{H}, \mathrm{SO}_{2}, \mathrm{CO}_{2}$, and $\mathrm{CN}$ (McCord et al. 1997). In addition abundant surface constituents of the Galilean moons are darker, non-ice materials (McCord et al. 1998), and, in the case of Europa, hydrated sulfuric acid (Carlson et al. 1999). Some of the darker materials could be organic compounds present at the time of the satellite formation or later delivered by cometary and meteoritic bombardment (Johnson et al. 2004).

$\mathrm{H}_{2} \mathrm{O}_{2}$ is formed by ion bombardment of pure water ice and such a process is believed to be responsible for its formation (Moore \& Hudson 2000; Gomis et al. 2004; Loeffler et al. 2006) with possible consequences for any biological activity in the liquid water possibly present below the ice in Europa and other satellites (Strazzulla et al. 2005). The experimental results of reactive ion implantation indicate that some molecular species observed on icy planetary surfaces (e.g. $\mathrm{CO}_{2}$ and hydrated sulfuric acid) could have been formed by implantation of reactive ions that populate the jovian (and others) magnetosphere and continuously bombard the surfaces of the embedded satellites. For a review of implantation experiments with reactive ions and the role they have to explain the presence of minor species on the jovian moons see Strazzulla (2011).

A further kind of experiments concerns the study of the synthesis of molecules at the interface between water ice and reactive surfaces such as carbonaceous or sulfurous solid materials. These have been stimulated by the fact that the surfaces of jovian moons are thought to contain significant amounts of the refractory (non-ice) materials, among which sulfurous materials (e.g. elemental sulfur; Carlson et al. 1999) and organic compounds (Johnson et al. 2004). Water ice has been deposited on different refractory carbonaceous materials, such as organic residues obtained after bombarding frozen hydrocarbon, hydrogenated amorphous carbon grains and asphaltite. A general finding is the formation of a noteworthy quantity of $\mathrm{CO}_{2}$ (Mennella et al. 2004). It has been suggested that this is the primary mechanism to explain the presence of carbon dioxide on the surfaces of the galilean satellites (Gomis \& Strazzulla 2005). On the contrary no evidence for an efficient synthesis of $\mathrm{SO}_{2}$ has been found after irradiation of water ice deposited on sulfur rich solids (Gomis \& Strazzulla 2008). Some implantation experiments have also been conducted in refractory targets such as silicates. In particular protons at energies from a few to 100's keV have been implanted in silicon and silicates to search for Si-H (Strazzulla et al. 1996; Djouadi et al. 2011) and O-H (Burke et al. 2011; Djouadi et al. 2011). The last results are timely and give different 
results: Burke et al. (2011) do not find evidence of O-H formation that is found by Djouadi et al. (2011) Thus the question of the formation of $\mathrm{H}_{2} \mathrm{O}$ in the lunar regolith via implantation of solar wind protons as a mechanism responsible for the significant $\mathrm{O}-\mathrm{H}$ absorption in recent spacecraft data, remains open.

\subsection{Colors}

A relevant effect of ion irradiation of solid materials is that the spectral slopes (colors) of the irradiated surfaces may significantly change. This has obvious consequences for the understanding of the "evolution" of solid surfaces in the Solar System, whose physico-chemical properties can be inferred from the observation of their reflectance spectra. Usually reddening and darkening of irradiated surfaces have been observed in the laboratory, although there are examples of "red" materials whose spectra turns grayish upon irradiation (see below). Although spectral slopes dramatically change when carbon bearing ices are irradiated in the laboratory, relevant effects are also observed for silicates and meteoritic samples.

It has been suggested that space weathering can be responsible for the mismatch between the spectra of the ordinary chondrites meteorites (OC) and the surface spectra of their presumed (S-type) asteroidal parent bodies. Ion irradiation (with $60 \mathrm{keV} \mathrm{Ar}^{++}$) of the ordinary chondrite Epinal (H5) produced darkening and reddening of the Vis-NIR spectra (Strazzulla et al. 2005). In Figure 1 diffuse reflectance spectra, normalized at $0.7 \mu \mathrm{m}$, of a sample of the Epinal meteorite before and after irradiation are compared with those observed for some S-type asteroids (433 Eros, 719 Albert, 1620 Geographos and 1916 Boreas; courtesy E. Dotto). It has been also estimated that the time-scale for this process is of the order of $10^{5}$ years at about $1 \mathrm{AU}$ (Astronomical Unit) from the Sun (Brunetto \& Strazzulla 2005). These results have been confirmed by Loeffler et al. (2009) that studied the effects of $4 \mathrm{keV} \mathrm{He}^{+}$irradiation on olivine and evidenced the correlation of the spectral changes with the amount of metallic iron formed by ion impact, consistent with the idea that space-weathering effects in the reflectance of olivine-bearing $\mathrm{S}$ type asteroids are due to the formation of metallic iron.

Visible and near-infrared reflectance spectra of TNOs, Centauri and other minor objects revealed a great variety of colors that are believed to be the result of competition between aging (e.g., cosmic ion irradiation that is simulated in the here discussed laboratory experiments), rejuvenating (e.g., impacts with meteorites, cryovolcanism, etc.) and evaporation processes (see e.g., Doressoundiram et al. 2002; Peixinho et al. 2004; Melita et al. 2009; Grundy 2009).

Irradiation experiments have been performed by irradiating different materials considered as templates of those possibly present on the surfaces of these objects. In particular frozen $(10-150 \mathrm{~K})$ ices (Brunetto et al. 2006) such as methane $\left(\mathrm{CH}_{4}\right)$, methanol $\left(\mathrm{CH}_{3} \mathrm{OH}\right)$ and benzene $\left(\mathrm{C}_{6} \mathrm{H}_{6}\right)$, asphaltite (a natural bitumen, Moroz et al. 2004) and polystyrene films deposited on an olivine (Kanuchova et al. 2010).

Irradiation of frozen ices evidence a strong reddening (Fig. 2) and darkening of the spectra, due to the formation of an organic (C-rich) refractory residue (Brunetto et al. 2006). Reddening increases with the amount of energy released 


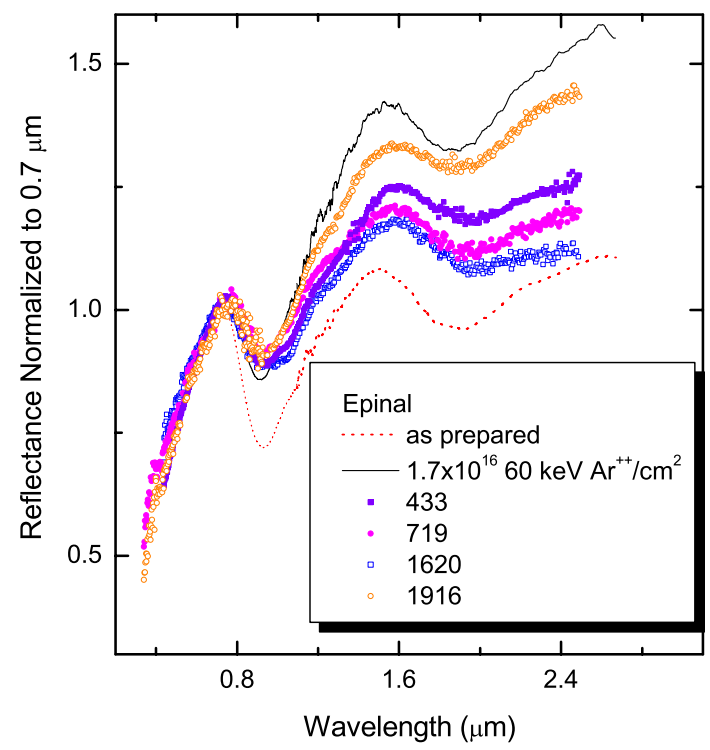

Fig. 1. Diffuse reflectance spectra, normalized at $0.7 \mu \mathrm{m}$, of a sample of the Epinal meteorite before and after irradiation with $60 \mathrm{keV}$ argon ions. The laboratory spectra are compared with those observed for some S-type asteroids (courtesy E. Dotto).

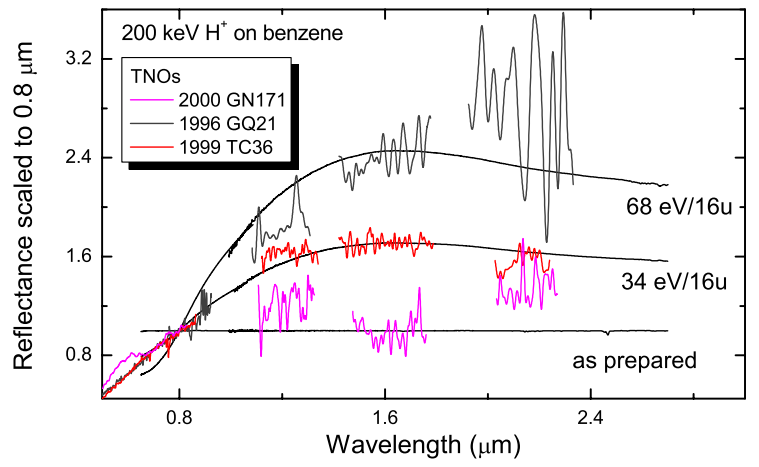

Fig. 2. Diffuse reflectance spectra, normalized at $0.8 \mu \mathrm{m}$, of organic material formed after irradiation of solid benzene with $200 \mathrm{keV}$ protons at different energy depositions $(\mathrm{eV} / 16 \mathrm{u})$. The laboratory spectra are compared with those observed for some Trans Neptunian Objects (courtesy of E. Dotto).

from the irradiating ions $\left(200 \mathrm{kev}^{+}\right.$in the shown example) to the target atoms, here given as $\mathrm{eV} / 16 \mathrm{u}$. The spectrum of the organic residue left over at higher temperatures reveals that it preserves the color also after the sublimation of the volatile species (both of the original target and formed by irradiation; Dalle Ore et al. 2011). 
Irradiation of such a solid residue as well as of natural red solids (e.g. asphaltite) produces darkening and flattening of the spectra (Moroz et al. 2004). Also originally transparent organic polymers such as polystyrene layers have been studied. Polystyrene, before irradiation, is a transparent polymer that, as ion fluence grows up, exhibits reddening and darkening. The reddening increases and reaches a maximum at a given fluence. Further irradiation produces further darkening but accomplished by a flattening of the spectrum (Kanuchova et al. 2010).

The laboratory spectra can be compared with those of several objects in the outer Solar System. As examples we can see a comparison of diffuse reflectance spectra, normalized at $0.8 \mu \mathrm{m}$, of organic material formed after irradiation of solid benzene with $200 \mathrm{keV}$ protons at different energy depositions (eV/16u) with those observed for some Trans Neptunian Objects (Fig. 2). It has been also demonstrated that using the fluxes of the relevant cosmic ion populations, the time scales necessary to reproduce the colors measured for weathered laboratory analogues is on the order of $10^{4}-10^{6}$ yrs i.e. compatible with the age of the surfaces of the small objects in the outer Solar System (e.g. Brunetto et al. 2006; Melita et al. 2009).

\section{Conclusion}

Ion irradiation plays a relevant role in the "space weathering" of solid materials on the airless bodies in the Solar System as well as in the interstellar medium. Here I have discussed some of the effects that have been studied in "simulation" experiments with a view to their astrophysical relevance. While a large amount of experiments have been performed, this research field still needs further efforts. As examples there are two broad areas that need to be investigated in more details:

- Irradiation of icy mixtures: although the synthesis of several specific, also complex, molecules has been reported by many groups, my feeling is that much more molecules are still to be revealed. In fact the detection techniques used up to now, essentially IR spectroscopy and mass spectrometry, are sensitive only to the synthesized species whose abundance is larger than several per mill with respect to the original species. Efforts have to be done to use more sensitive techniques to evidence the formation of additional complex molecules and/or fragments that could be of primary relevance for e.g. Astrobiology.

- Silicates: existing (e.g. Itokawa, Messenger) or foreseen (e.g. Bepicolombo) space missions to Mercury and other rocky objects evidence the role of space weathering. Still data on e.g. sputtering induced secondary ion emission by low energy, multi charged ions simulating solar wind are scarce. In particular the "potential" sputtering induced by multi charged ions (e.g. Meyer et al. 2010) and the role that emitted atoms and/or ions have to substain the tenuous atmospheres of Mercury and the Moon have to be investigate in detail. 


\section{References}

Baragiola, R.A., 2004, RSLTS, 362, Issue, 1814, 29

Brown, W.L., et al., 1978, PRL, 40, 1027

Brunetto, R., \& Strazzulla, G., 2005, Icarus, 179, 265

Brunetto, R., et al., 2006, ApJ, 644, 646

Burke, D.J., et al., 2011, Icarus, 211, 1082

Carlson, R.W., Johnson, R.E., \& Anderson, M.S., 1999, Science, 286, 97

Dalle Ore, C., et al., 2011, A\&A, 533, A98

Djouadi, Z., et al., 2011, A\&A, 531, 96

Doressoundiram, A., et al., 2002, AJ, 124, 2279

Dukes, C.A., et al., 2011, Icarus, 212, 463

Hudson, R.L., et al., 2008, in "The Solar System Beyond Neptune", ed. M.A. Barucci, H. Boehnhardt, D.P. Cruikshank \& A. Morbidelli (University of Arizona Press, Tucson), 507

Gomis, O., \& Strazzulla, G., 2005, Icarus, 177, 570

Gomis, O., \& Strazzulla, G., 2008, Icarus, 194, 146

Gomis, O., et al., 2004, Pl. Sp. Sci., 52, 371

Grundy, W.M., 2009, Icarus, 199, 560

Johnson, R.E., et al., 1998, Geo. Res. Lett., 25, 3257

Johnson, R.E., 2002, in Atmospheres in the Solar System: Comparative Aeronomy, Geophysical Monograph, 130, 203

Johnson, R.E., et al., 2004, in: Jupiter: Planet, Satellites, and Magnetosphere, ed. F. Bagenal, W. McKinnon \& T. Dowling (Cambridge Univ. Press, Cambridge, UK), 485

Kanuchova, et al., 2010, A\&A, 517, A60

Loeffler, M.J., Dukes, C.A., \& Baragiola, R.A., 2009, JGR, 114, CiteID, E03003

Loeffler, M.J., et al., 2006, Icarus, 80, 265

McCord, T.B., et al., 1997, Science, 278, 271

McCord, T.B., et al., 1998, JGR, 103, 8603

Melita, M.D., Strazzulla, G., \& Bar-Nun, A., 2009, Icarus, 203, 134

Mennella, V., Palumbo, M.E., \& Baratta, G.A., 2004, ApJ, 615, 1073

Milillo, A., et al., 2011, JGR, 116, CiteID, A07229

Moore, M.H., \& Hudson, R.L., 2000, Icarus, 145, 282

Moroz, L., et al., 2004, Icarus, 170, 214

Peixinho, N., et al., 2004, Icarus, 170, 153

Strazzulla, G., 2011, NIMB, 269, 842

Strazzulla, G., Brucato, J.R., Palumbo, M.E., \& Satorre, M.A., 1996, NIMB, 116, 289

Strazzulla, G., et al., 2005, Astrobiology, 5, 612

Vervack, Jr., R.J., et al., 2010, Science, 329, 672

Ziegler J.F., Biersack, J.P., \& Ziegler, M.D., 2008, The stopping and range of ions in solids (New York: Pergamon Press) 